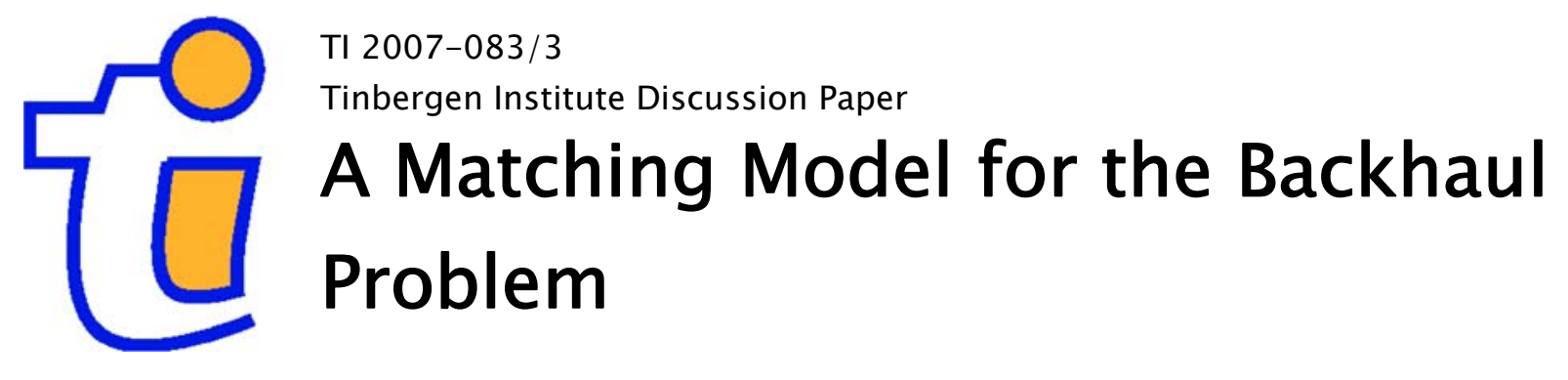

Erhan Demirel

Jos van Ommeren

Piet Rietveld

Dept. of Spatial Economics, Vrije Universiteit Amsterdam, and Tinbergen Institute. 


\section{Tinbergen Institute}

The Tinbergen Institute is the institute for economic research of the Erasmus Universiteit Rotterdam, Universiteit van Amsterdam, and Vrije Universiteit Amsterdam.

Tinbergen Institute Amsterdam

Roetersstraat 31

1018 WB Amsterdam

The Netherlands

Tel.: $\quad+31(0) 205513500$

Fax: $\quad+31(0) 205513555$

Tinbergen Institute Rotterdam

Burg. Oudlaan 50

3062 PA Rotterdam

The Netherlands

Tel.: $\quad+31(0) 104088900$

Fax: $\quad+31(0) 104089031$

Most TI discussion papers can be downloaded at http:/ /www.tinbergen.nl. 


\title{
A MATCHING MODEL FOR THE BACKHAUL PROBLEM
}

\author{
Erhan Demirel, Jos van Ommeren, Piet Rietveld
}

Vrije Universiteit, Department of Spatial Economics,

De Boelelaan 1105, 1081 HV Amsterdam, The Netherlands

edemirel@feweb.vu.nl,jommeren@feweb.vu.nl, prietveld@feweb.vu.nl

December 19, 2007

KEYWORDS: Backhaul Problem, Imperfect Information, Matching Models, Search Costs

\begin{abstract}
The 'backhaul problem' is characterized by an imbalance in transport flows between locations. In a perfectly competitive framework, the price of transport from low demand locations to high demand locations, the so-called backhaul price, drops to zero when the imbalance is sufficiently large. However, this result is inconsistent with empirical observations for many transport markets (e.g. taxi, inland and maritime shipping markets). In this paper, we develop a matching model to address this inconsistency. We argue that carriers' search time to locate customers may play an important role in the determination of prices. We demonstrate that carriers are compensated for the time they search for customers. This implies positive backhaul prices. The matching model is numerically applied to the inland navigation shipping market in the Rhine river area in Western-Europe. We find that backhaul prices are substantial.
\end{abstract}

This paper has been written in the context of the 'Vulnerability, Adaptation and Mitigation'-project (VAM) and the 'Climate changes Spatial Planning'-project. We thank NWO for financial support. 


\section{Introduction}

The 'backhaul problem' is a well-known phenomenon in transport economics, both in freight and passenger transport studies. It refers to the situation where the volume of transported goods or persons is not in balance between two (or more) locations, which means that transport flows are mainly in one (or more) dominant direction(s). The 'backhaul problem' is one of the classical research problems, going back to at least the disscussion of 'joint costs' by Pigou and Taussig (1913).

Imbalance in freight transport flows is extremely common (see e.g. Wilson, 1987). In the current study we aim to study the effect of imbalance on price formation, so one must know the degree of imbalance at the level of the individual carrier, which is difficult to observe. The imbalance at a more aggregate level has been extensively studied however. In particular, the imbalance in flows at the national level using annual observations has been studied by a large number of studies in the trade economics literature and has been shown to be substantial (see e.g. Lee et al., 2006). Most likely, the imbalance is much more pronounced for transport flows between smaller regions and shorter periods of observation (due to timevariation in demand). At the level of carriers, imbalance may even be more pronounced, because carriers are frequently specialised in a certain type of freight which generates additional imbalance. For example, transport carriers which are specialised in the transport of edible oils are generally not allowed to transport other kinds of (nonedible) freight.

At first sight it may seem that in the passenger market the backhaul problem is absent, as nearly all passengers return to their location of origin. However, there is a large time variation in demand which induces low load factors and therefore a backhaul problem in public transport, and also in the taxi market.

Theoretical contributions to the backhaul literature use perfect-competition models that are based on complete information (see e.g. Felton, 1981). ${ }^{1}$ To simplify the framework, these studies examine transport between two locations. Theories based on perfect competition predict that above a minimum degree of imbalance in demand, the quantity transported from location L, with low demand for transport, is less than location $H$, with high demand for transport, and the price for transport from the low demand location to the high demand

\footnotetext{
${ }^{1}$ Rietveld and Roson (2002) model the price setting of a public transport monopolist, but also in this study complete information is assumed. 
location, the so-called backhaul price, will drop to zero. This is intuitive because carriers are indifferent between returning with or without backhaul. ${ }^{2}$ This means that the cost of transport from one location to the other is fully borne by the high demand location's customers. ${ }^{3}$ This cost is equal to the round trip cost of transport. The type of equilibrium where the quantities transported are not the same in both directions will be called throughout this paper the Imbalanced Equilibrium.

Another equilibrium may arise, which we will call throughout this paper the Balanced Equilibrium. The quantities transported then are exactly the same in both directions. According to the competitive model, in this equilibrium the carriers' value of returning with backhaul exceeds the value of returning without backhaul and all carriers return with backhaul. Fronthaul prices will be higher than the backhaul price due to the difference in demand, but the backhaul price will be positive.

A summary of these two types of equilibria is given in Table 1 , where $Q_{i}$ and $p_{i}$ $(i=H, L)$ represent quantity transported and the price of transport in location $i$ respectively. In this setting, there is no third equilibrium where $Q_{H}>Q_{L}$, so some of the carriers return empty from location $L$, and, at the same time, $p_{H} \geq p_{L}>0$.

Table 1: Summary of the two Equilibria for the competitive model.

\begin{tabular}{cccc}
\hline & Quantities Transported & Prices & Interpretation \\
\hline Balanced Equilibrium & $Q_{H}=Q_{L}$ & $p_{H}>p_{L}>0$ & \\
Imbalanced Equilibrium & $Q_{H}>Q_{L}$ & $p_{H}>\boldsymbol{p}_{\boldsymbol{L}}=\mathbf{0}$ & Backhaul Problem \\
\hline
\end{tabular}

It is not difficult to give a large range of anecdotal examples for the taxi market that describe the backhaul problem that depart from the predictions of the competitive model. In the Netherlands, cab drivers and customers are allowed to bargain about the price (up to a maximum), but in our experience, cab drivers never offer trips at even close to zero prices, even if the taxi will go anyway in the direction the customer wishes to go. One may also examine freight prices, which are publicly available, for numerical evidence. For example, in the maritime sector, the freight prices for 1 TEU container of plastic bags, from Shanghai to San Francisco is $\$ 2,065$, whereas the backhaul price is $\$ 1,111$. So the backhaul price is

\footnotetext{
${ }^{2}$ This result is the classical result as featured in transport economics textbooks such as Boyer (1998).

${ }^{3}$ For convenience, transport-demanding customers are called customers in the text. 
roughly $50 \%$ less than the fronthaul price, but it is far from zero. ${ }^{4}$ As is well known, the imbalance between merchandise goods flows between China and the U.S. is large (in value terms, the flow from China is 4 times that of the return flow (see WTO, 2005). Nevertheless the backhaul price for the return trip from the U.S. to China is considerable.

In the inland navigation market, Jonkeren et al. (2007) show that backhaul prices (from Germany to the Netherlands) are $76 \%$ of the fronthaul prices although the quantity transported from the fronthaul location (the Netherlands) exceeds the quantity transported from the backhaul location (Germany) by at least 50\% (Statistics Netherlands, 2007). ${ }^{5}$ Nevertheless, backhaul prices are far from close to zero.

The competitive model assumes that customers and carriers possess complete information about each other's (future) location in the market. Customers and carriers find each other immediately. This implies the absence of market friction and of search time for carriers as well as for customers. In many transport markets, search time for customers is an obvious cost component, not only in the taxi market where taxis either cruise or passively wait for customers (see Arnott, 1996), but also in the inland shipping market (see Meelker, 2006). One might add exogenous search times in the standard competitive model in order to arrive at positive backhaul prices, but this does not do justice to the fact that search times are endogenous, (see Arnott, 1996). In the matching model we introduce in section 2, we show that the endogenously determined carrier's expected search time is a possible cause for positive backhaul prices. ${ }^{6}$

Matching models are nowadays popular to analyse markets where agents are searching for each other and face a certain difficulty to find each other and form a match. This kind of models are now standard in the labour market economics literature (see e.g. Pissarides, 2000), but are also used in housing economics (see e.g. Wheaton, 1990). For examples of matching models to study the taxi market, see Lagos (1996) and Arnott (1996). The matching model is also well applicable in many freight transport markets, as carriers and customers search for

\footnotetext{
${ }^{4}$ These numbers are taken from www.freight-calculator.com in November, 2007. Note that in this example, insurance, additional fuel and handling costs are close to zero, so we may interpret the freight prices as prices net of insurance, additional fuel and handling costs.

${ }^{5}$ Plausibly, as argued above, the imbalance at the level of the carriers is substantially higher due to restrictions on the type of freight and variation in demand over time etc.

${ }^{6}$ Other reasons for positive backhaul prices may be found in increased fuel costs for transport with freight and waiting time due to loading up and unloading. These costs could be added exogenously to both the competitive and the matching model. However, we argue that these are not the only causes of positive backhaul prices and cannot explain the examples in the main text.
} 
each other and have difficulty to find each other. As argued above, this difficulty is due to a combination of spatial and time variation in demand and supply.

In the current paper, we introduce in Section 2 a two-location transport framework which incorporates a matching model to study the backhaul problem. In Section 3 we analyse the backhaul problem numerically, with input values chosen from the inland navigation market on the Rhine river in Western-Europe. Section 4 concludes.

\section{The Matching Model}

\subsection{The matching function}

Suppose that a fixed number of identical risk-neutral customers with a demand for transport are located in locations $H$ and $L$. We define the numbers of customers located at each side by $N_{i}$ where $i=H, L$. We allow for a difference in demand between location $H$ and location $L$. Hence, the number of customers in one location exceeds the number of customers in the other location. We choose (arbitrarily) location $H$ to be the location with high demand for transport, and $L$ to be the location with low demand for transport, so $N_{H} \geq N_{L}>0{ }^{7}$ For convenience, other exogenous parameters are assumed to be identical for both locations.

Each customer aims to have one good transported by identical risk-neutral carriers to the opposite side. We assume that a customer withdraws from the market after it has found a carrier that is willing to transport the good to the other side and is immediately replaced by a new customer, so the number of customers remains constant over time. ${ }^{8}$ A carrier may at most transport one good, so it is either full or empty.

We assume that customers and carriers have to search for each other due to imperfect information about each other. It is assumed that given search, customers and carriers contact each other according to a well-defined contact function. Carriers and customers are only able to contact each other when they are in the same location $L$ or $H .{ }^{9}$ The contact function in location $i$ specifies then the number of contacts taking place in location $i$ during a time period as a function of the number of searching carriers and customers in that location.

\footnotetext{
${ }^{7}$ The case that $N_{L}=0$ can be received upon request from the authors.

${ }^{8}$ This is an easy way of modeling freight demand without having to worry about the exact production process of the goods transported.

${ }^{9}$ We restrict the search technology in this way to avoid mathematical complexities, see e.g. Pissarides (1994). Even though this assumption about the contact function might be restrictive for some transport markets, it is unlikely to affect our main conclusions.
} 
Given a contact, the customer and the carrier bargain about the freight price. If they agree on a price, which we later on show to occur with probability one, the customer and carrier are matched. Hence, the matching function is identical to the contact function. When a carrier and a customer are matched, the customer pays the carrier the agreed freight price, the cargo is loaded up and the carrier is obliged to transport the good towards the other location.

In the current paper, we focus on the steady state. We assume that transportation is costly, as it takes time. At every moment in time, there are $C_{M}$ carriers on their way transporting between locations with or without freight. We denote $C_{S i}$ as the number of searching carriers at location $i$. The total number of carriers in the market $C$ is then equal to $C_{M}+C_{S H}+C_{S L}$. In the steady state, the number of carriers transporting in both directions must be equal, so $C_{M} / 2$ carriers transport in every direction.

We define $u_{i}=C_{S i} / C$ as the number of carriers that search in location $i$ relative to the total number of carriers and $v_{i}=N_{i} / C$ as the number of customers relative to the total number of carriers. ${ }^{10}$ Therefore $0<u_{i}<1$ and $0<v_{i}<\infty$. We introduce the variable $\theta_{i}=v_{i} / u_{i}$, which can be interpreted as a measure of market tightness in $i$. It can be easily seen that $\theta_{i}$ equals $N_{i} / C_{S i}$, the number of customers divided by the number of searching carriers in location $i$.

The (continuous) contact function, denoted as $m$, defines the number of contacts between searching carriers $C_{S i}$ and the number of customers, $N_{i}$, per (infinitely small) period. This function can therefore be written as $m\left(C_{S i}, N_{i}\right)$ and is assumed to be increasing in both arguments. The function is assumed to have non-decreasing returns to scale in its arguments, such that $m\left(\omega C_{S i}, \omega N_{i}\right)=\alpha \omega m\left(C_{S i}, N_{i}\right)$, where $\alpha$ is a given constant larger than or equal to one. This assumption means that contacting may become easier when markets are more dense. $^{11}$

We define $q_{i}$ as the customers contact rate, so the rate at which a customer contacts a searching carrier in $i$. It follows that:

$$
q_{i}=\frac{m_{i}}{N_{i}}=\frac{m\left(u_{i} C, v_{i} C\right)}{v_{i} C}=\alpha m\left(\frac{u_{i}}{v_{i}}, 1\right)=\alpha m\left(\frac{1}{\theta_{i}}, 1\right) \quad \text { with } \alpha \geq 1
$$

\footnotetext{
${ }^{10}$ Note that ' $u$ ' may be interpreted as unemployed carriers, and ' $v$ ' may be interpreted as the number of customers with a vacancy.

${ }^{11}$ Note that when $\alpha=1$ the contact function has the constant returns to scale property. For the literature on constant and increasing return to scale in the labour market, see Warren (1996). Increasing returns to scale is a common property of spatial search models, so it seems natural to allow for this property.
} 
This implies that $q_{i}$ is a negative function of $\theta_{i}$, whereas $\theta_{i} q_{i}$ is a positive function of $\theta_{i}$. Given $\theta_{i}$ and $q_{i}$, the carrier's contact rate at which a searching carrier contacts a customer is determined and defined by $\theta_{i} q_{i}$. We emphasize that the contact rates, $q_{i}$ and $\theta_{i} q_{i}$, are both endogenously determined.

\subsection{Bellman Equations}

Carriers are in two possible states: they are either searching at a certain location $i$ or moving/transporting between two locations (with or without freight). For further modeling purposes, a monetary asset value is assigned to be in one of these two states. We define $S_{i}$ as the asset value of searching in $i$, and $M_{i}$ as the asset value of moving/transporting from location $i$ to location $j$. It is convenient to define $M_{i}$ at the moment of departing from location $i .^{12}$ Note that $M_{i}$ may be positive or negative. In the latter case, it is more convenient to interpret $M_{i}$ as the monetary value of the obligation to transport from location $i$ to location $j$.

To derive the asset value of searching $S_{i}$, we define $s c$ as the carriers' instantaneous search costs per unit of time, and $p_{i}$ as the price received to transport a good from location $i$ to the opposite side. The discount rate is denoted as $r$ per unit of time. The following Bellman equation defines then the value of $S_{i}$ :

$$
r S_{i}=-s c+\theta_{i} q_{i}\left(p_{i}+M_{i}-S_{i}\right) .
$$

The interpretation of (1) is straightforward (see, similarly, Mortensen and Pissarides, 1999). The left-hand-side is the return of searching $r S_{i}$. The right-hand-side is equal to the cost of searching, $-s c$, plus the expected gain of finding a customer and transporting the good to the other side, which is equal to $\theta_{i} q_{i}\left(p_{i}+M_{i}-S_{i}\right)$. So the expected gain is equal to the product of $\theta_{i} q_{i}$, the rate at which a carrier finds a customer, and $p_{i}+M_{i}-S_{i}$, which is the surplus a carrier gains from a match with the customer. The surplus of the match is equal to the received price $p_{i}$ of transport to the other location plus the asset value of moving $M_{i}$, minus the asset value of searching $S_{i}$.

The carriers' cost of transporting the good is assumed to be independent of whether the carrier transports freight and is proportional to the time it takes to transport the good. We

\footnotetext{
${ }^{12}$ Note that the value of transporting depends on where the carrier is between $H$ and $L$. In the current model, we allow for this complication, but we need only to define the asset value at the moment of departing from $H$ and $L$. 
define $m c$ as the cost of transporting/moving per unit of time. The duration of the trip is stochastically determined. We assume that the average duration of a round trip is distributed with a mean equal to $1 / \lambda$. So $\lambda$ can be interpreted as the average speed at which a carrier moves between locations. As we have assumed that customers and carriers are risk neutral, all decisions made by carriers will not depend on other properties of the distribution than its mean. Hence, for convenience, we will assume that the duration of a round trip is exponentially distributed. In this case, the duration of a single trip is exponentially distributed with mean equal to $1 /(2 \lambda)$. Consequently, $2 \lambda$ can also be interpreted as the rate of arriving at the other location.

Given these assumptions, the following equation defines the asset value of $M_{i}$ :

$$
r M_{i}=-m c+2 \lambda\left(\max \left(S_{j}, M_{j}\right)-M_{i}\right), \quad \text { for } j \neq i .
$$

Equation (2) defines the return on the asset of moving, $r M_{i}$. It consists of the immediate cost of moving, $-m c$, plus the expected gain of arriving at the other location. This gain is equal to the rate of arriving at the other location, $2 \lambda$, multiplied with the net value of arriving at the other location $j$, defined by $\max \left(S_{j}, M_{j}\right)-M_{i}$, where $j \neq i$. Note that this net value depends on the maximum value of two types of strategies in the other location (searching or moving) because carriers may choose then between two strategies: searching for freight or moving without freight to the other location. ${ }^{13}$

When a customer searches for a carrier to transport its good, the customer attaches an asset value $V_{i}$ to being this state, (which can also be interpreted as the value of being in this kind of market). When matching occurs, the customer receives immediately price $J$ for the good, and pays the price $p_{i}$ for transport. ${ }^{14}$ There is a per period cost of search $\kappa$ to find a carrier. ${ }^{15}$ The following Bellman equation defines then $V_{i}$ :

$$
r V_{i}=-\kappa+q_{i}\left(J-V_{i}-p_{i}\right)
$$

\footnotetext{
${ }^{13}$ In equilibrium, it can never be optimal for a carrier to search first and move to the other side after a while without freight. As market circumstances do not change in our model, moving to the other side without freight may only occur immediately after arriving.

${ }^{14}$ Note that one may also assume that the customer receives $\mathrm{J}$ the moment that the good arrives at the other side. As travel durations are exogenous, this gives the same results.

${ }^{15}$ This cost may be interpreted as the combined cost of search and storage. 
The customers' return on searching $r V_{i}$ is thus equal to the search cost, $-\kappa$, plus the expected gain of finding a carrier $q_{i}\left(J-V_{i}-p_{i}\right)$. This expected gain is the rate $q_{i}$ multiplied by the surplus $J-V_{i}-p_{i}$. This surplus is equal to the revenue $J$ for selling the product minus the price $p$ paid for transport, and minus the loss of asset $V_{i}$, as the customer leaves the market. Solving for $V_{i}$ gives $V_{i}=\left(q_{i}\left(J-p_{i}\right)-\kappa\right) /\left(r+q_{i}\right)$.

We assume free entry of carriers at no cost. ${ }^{16}$ The number of carriers in the market, $C$, is therefore endogenously determined. In particular, we assume that carriers may only enter and leave the market in $H$ (the location with high demand) that this is the location with the highest value of search, so $S_{H} \geq S_{L}$, and that carriers that enter the market have to make at least one round trip. This means that entry and exit of carriers at location $H$ will occur until $S_{H}=0$ and $S_{L} \leq 0$.

We will impose now a restriction such that the market exists. We suppose that $J+\kappa / r$ exceeds $m c /(r+\lambda)$. This condition is intuitive. It simply states that the transport cost must be less than the benefits of transport (which equals $J$ plus the cost of search forever).

When the transport market exists (so goods are transported), a necessary implication is that in location $H$ or $L$ the value of searching strictly exceeds the value of moving to the other side without freight (for a proof see Appendix A). By the free-entry condition $S_{H}=0$ and $S_{L} \leq 0$, and (2) it follows that $S_{H}>M_{H}$. One of the following two equilibria will then occur: ${ }^{17}$

$\begin{array}{llll}\text { the Imbalanced Equilibrium: } & S_{H}>M_{H} & \text { and } & S_{L}=M_{L} ; \\ \text { the Balanced Equilibrium: } & S_{H}>M_{H} & \text { and } & S_{L}>M_{L} .\end{array}$

Both equilibriums may arise. For example, in the extreme case that $N_{H}=N_{L}$, it appears that the Balanced Equilibrium will occur. ${ }^{18}$ When $N_{H}>N_{L}$, one may have either the Balanced or Imbalanced Equilibrium. Intuitively, when $N_{H}$ is close to $N_{L}$, the Balanced Equilibrium will occur, and when $N_{H}$ is much greater than $N_{L}$, then the Imbalanced equilibrium will arise. As emphasized in the introduction, we are in particular interested in the Imbalanced Equilibrium.

Note that in both equilibria, $S_{H}$ exceeds $M_{H}$, so all carriers in $H$ search for freight (and never return empty to $L$ ). This means that the fraction of carriers that arrive in $\mathrm{H}$ and search

\footnotetext{
${ }^{16}$ This is a natural long-run assumption in the absence of sunk costs.

${ }^{17}$ Note that we assumed that $N_{L}>0$. In the case that $N_{L}=0$, then $S_{L}<M_{L}$ and an equilibrium with only one-way transport will arise.

${ }^{18}$ This is easy to see, as due to symmetry, market outcomes in $L$ must be the same as in $H$. 
for freight, denoted by $\mu_{H}$, is equal to one $\left(\mu_{H}=1\right)$. In the Imbalanced Equilibrium, carriers in $L$ are indifferent between searching for freight or returning empty to $H$. Therefore a strictly positive fraction, denoted as $1-\mu_{L}$ of carriers, will return empty from $L$ to $H$. The variable $\mu_{L}$ represents the fraction of carriers that arrive in $L$ and search in $L$ for freight. In the Balanced Equilibrium, $\mu_{L}=1$ and $S_{L}>M_{L}$. In the Imbalanced Equilibrium, $0<\mu_{L}<1$ and $S_{L}=M_{L}$. Hence the following condition holds:

$$
\left(\mu_{L}-1\right)\left(S_{L}-M_{L}\right)=0 \text {. }
$$

We emphasize that it is the Imbalanced Equilibrium, which is of main empirical interest, as in this equilibrium carriers can be said to have a 'backhaul problem'.

\subsection{Price determination in the long run}

Given equations (1) to (4), and the free-entry assumption which implies that $S_{H}=0$, we obtain, as shown in Appendix B, the following two price equations:

$$
\begin{aligned}
& p_{H}=\frac{s c}{\theta_{H} q_{H}}+\frac{m c}{r+2 \lambda}-\frac{2 \lambda}{r+2 \lambda} S_{L}, \\
& p_{L}=\frac{s c}{\theta_{L} q_{L}}+\frac{m c}{r+2 \lambda}+\frac{\theta_{L} q_{L}+r}{\theta_{L} q_{L}} S_{L} .
\end{aligned}
$$

Note that $s c / \theta_{i} q_{i}$ can be interpreted as expected search costs. Consequently, freight prices in both locations are equal to the sum of the expected search cost, the discounted one-way cost of transporting the good and a term which is, when the discount rate $r$ is small, approximately equal to $-S_{L}$ in the high demand location and equal to $S_{L}$ in the low demand location. Recall that $S_{L} \leq 0$. Consequently, freight prices in the high demand location $p_{H}$ exceed the prices in the low demand location $p_{L}$. Note that the exact value of $S_{L}$ depends on the type of equilibrium. It appears that $S_{L}=-m c /(r+2 \lambda)$ when the equilibrium is Imbalanced and $S_{L}>-m c /(r+2 \lambda)$ when the equilibrium is Balanced.

Interpretation of the above equations is facilitated by assuming that $r / \lambda$ approaches zero. Note that $r$ and $\lambda$ are both strictly positive, so this implies that $\lambda$ is much larger than $r$. 
This assumption is reasonable as $r / \lambda$ is very small in practice. ${ }^{19}$ Given this assumption, $p_{L}+p_{H}=\frac{s c}{\theta_{L} q_{L}}+\frac{s c}{\theta_{H} q_{H}}+\frac{m c}{\lambda}$. So the sum of the prices in $H$ and $L$ is equal to the sum of the round trip costs and the carriers' search costs. ${ }^{20}$ Then, (5) and (6) imply that:

$$
p_{H} \leq \frac{s c}{\theta_{H} q_{H}}+\frac{m c}{\lambda} \quad \text { and } \quad p_{L} \geq \frac{s c}{\theta_{L} q_{L}}
$$

where equality holds in the Imbalanced Equilibrium. Hence, in the Imbalanced Equilibrium, the price in the low demand location, the backhaul price, is a compensation for a carrier's expected search costs. Only when $\theta_{L} q_{L}$ approaches infinity, so the expected search time in the low demand location approaches zero, then $p_{L}=0$. However, after section 2.4, we formulate Theorem 1 in section 2.5, and in its proof in Appendix $\mathrm{C}$ we show that $\theta_{L} q_{L}$ is finite. Therefore backhaul prices $p_{L}$ will always be positive.

\subsection{Steady-state equilibrium stocks and flows}

In steady state, the outflow of carriers from a location equals the inflow of carriers into that location. Hence, at $i$, it must hold that $\theta_{i} q_{i} u_{i}=\mu_{i} \lambda\left(1-u_{i}-u_{j}\right)$, for $i \neq j$. We solve now for the number of searching carriers in both locations, $u_{i}(i=L, H)$, so:

$$
u_{i}=\frac{\lambda \mu_{i}}{\theta_{i} q_{i}+\lambda\left(\mu_{i}+\mu_{j} \frac{\theta_{i} q_{i}}{\theta_{j} q_{j}}\right)} \quad \text { for } j \neq i \text {, }
$$

where $\mu_{H}=1$ and $0<\mu_{L} \leq 1$.

Hence, the number of searching carriers in $i$ depends on the speed $\lambda$, and $\theta_{i} q_{i}$, which is the inverse of carriers' average search time in location $i$, the fraction of carriers that return with freight in $i$ as well as $j, \mu_{i}$ and $\mu_{j}$ respectively, as well as the search time in $i$ relative to the search time $j$. In the case that $\theta_{i} q_{i}$ approaches infinity (expected search time in $i$ is zero), then $u_{i}$ approaches zero. This is the situation assumed in the competitive model.

\footnotetext{
${ }^{19}$ For example, for the numerical example later on, it seems reasonable to assume that $\mathrm{r}$ is 0.05 and $\lambda$ is 50 , so $r / \lambda$ is equal to 0.001 .

${ }^{20}$ Clearly, when the interest rate approaches zero, carriers have no intrinsic preference for the presence. So for each round trip they only care about the sum of the freight prices and the sum of the costs.
} 


\subsection{Bargaining and conditions for positive backhaul prices}

Given a contact, the carrier and the customer are assumed to negotiate a freight price $p_{i}$ according to a Nash-bargaining rule (see Binmore et al. (1986) ${ }^{21}$. It is assumed that:

$$
p_{i}=\arg \max \left(M_{i}-S_{i}+p_{i}\right)^{\beta}\left(J-V_{i}-p_{i}\right)^{l-\beta},
$$

where $\beta$ measures the bargaining power of the carriers, and $1-\beta$ measures the bargaining power of the customer $(O<\beta<1)$. The first-order condition with respect to $p_{i}$ is then

$$
\beta\left(J-V_{i}-p_{i}\right)=(1-\beta)\left(M_{i}-S_{i}+p_{i}\right)
$$

Equations (1) and (3) imply that $J-V_{i}-p_{i}>0$ and $M_{i}-S_{i}+p_{i}>0$. Since customers and carriers are identical, it follows that every contact will result, after bargaining, in a match.

For the Imbalanced Equilibrium, we derive now the backhaul price from the low demand location, $p_{L}$. In the Imbalanced Equilibrium, $M_{L}=S_{L}$ holds. Using (3) and (10):

$$
\beta\left(J-\frac{q_{L}\left(J-p_{L}\right)-\kappa}{r+q_{L}}-p_{L}\right)=(1-\beta) p_{L}
$$

Solving for $p_{L}$ :

$$
p_{L}=\frac{\beta(r J+\kappa)}{r+(1-\beta) q_{L}}
$$

We are now able to formulate Theorem 1, which holds for both the Balanced and Imbalanced Equilibrium.

\section{Theorem 1.}

In a matching model for a two-location market the backhaul price $p_{L}$ will be strictly positive, under the condition that the discount rate is strictly positive $(r>0)$.

\footnotetext{
${ }^{21}$ This bargaining rule has become standard in the labour market and housing market literature, see e.g. Wheaton (1990). 
For the Balanced Equilibrium, this result can be given without further proof, as in this Equilibrium $S_{L}>M_{L}$ holds, which implies that search in the low demand location, $L$, has a higher value than returning empty. This must be induced by a positive compensation for the waiting costs, which they receive as the strictly positive backhaul price $\left(p_{L}>0\right)$. For the Imbalanced Equilibrium, the proof of Theorem 1 is given in Appendix C. In this appendix it is also proven that $\theta_{L} q_{L}$ is finite.

Similar to the competitive model, we may now construct Table 2, which summarizes the main result of our matching model. It presents the quantities transported and the prices under the two equilibria for the matching model. The quantities transported follow from the definition of the two equilibria. The prices follow from the proof of Theorem 1 in Appendix C.

Table 2: Summary of the two Equilibria for the two-location matching model.

\begin{tabular}{ccc}
\hline & Quantities Transported & Prices \\
\hline Balanced Equilibrium & $Q_{H}=Q_{L}$ & $p_{H} \geq p_{L}>0$ \\
Imbalanced Equilibrium & $Q_{H}>Q_{L}$ & $p_{L}>0$ \\
\hline
\end{tabular}

\section{Numerical Analysis}

\subsection{Calibration of the model (the reference case)}

In order to perform a numerical analysis, we assume a functional form for the matching function $m$. We use a Cobb-Douglas function $m(x, y)=\phi x^{\gamma} y^{\delta}$, where $\gamma+\delta \geq 1$, which has the property of nondecreasing returns to scale. Note that $\phi$ is a scale parameter of friction. This implies that

$$
q_{i}=\phi \theta_{i}^{-\gamma} N_{i}^{\gamma+\delta-1}
$$

It has been established above that the prices depend on the expected search cost of carriers. In our model, these costs depend, among others, on the degree of search friction in the market, represented by $\phi$, the value of the good $J$, the imbalance in demand between the two 
locations, represented by the number of customers $N_{H}$ and $N_{L}$ and on the speed $\lambda$. For numerical analysis we explore consequences of varying some of these key parameters. Numerical values for the exogenous parameters $s c, m c, \lambda, \kappa, r$ and $J$ have been chosen for the market of inland navigation carriers which predominantly transport freight between the Netherlands and Germany on the Rhine River (see Jonkeren et al. (2007) and Table 3 for details). The time period is taken as one year. The values of $\phi, \gamma$ and $\delta$, which are difficult to observe, are obtained by calibrating the model using information on search times of customers and carriers both in $H$ and $L . \phi$ is set to 30. The Cobb-Douglas parameters $\gamma$ and $\delta$ are set to $\gamma=0.6$ and $\delta=0.6$. Note that $\gamma+\delta>1$, which implies 'slightly' increasing returns to scale. A $100 \%$ increase in the number of customers and searching carriers implies then an increase in the number of matches of $130 \%,\left(2^{0.6} * 2^{0.6}=2.30\right)$.

Table 3: Exogenous parameters

\begin{tabular}{lll}
\hline Parameter & Value & Description \\
\hline$s c$ & $€ 150,000$ & Annual carriers' search cost \\
$m c$ & $€ 250,000$ & Annual carriers' cost of moving/transporting \\
$\lambda$ & 50 & $\begin{array}{l}\text { Annual trip speed measured in round trips per year. We assume a return trip } \\
\text { duration of ca. 1 week }\end{array}$ \\
$\kappa$ & $€ 25,000$ & Annual customers' search cost \\
$r$ & 0.05 & Annual interest rate \\
$N_{H}$ & 400 & Number of customers located in $H$ \\
$N_{L}$ & 100 & Number of customers located in $L$ \\
$J$ & $€ 100,000$ & Received price by a customer for the good that is transported \\
$\beta$ & 0.5 & Bargaining power parameter \\
$\gamma$ & 0.6 & Parameter of Cobb-Douglas matching function \\
$\delta$ & 0.6 & Parameter of Cobb-Douglas matching function \\
$\phi$ & 30 & Friction parameter \\
\hline
\end{tabular}

Using the model of section 2, the values of the endogenous variables are given in Table 4 and Table 5 with a description given for every variable. Note that for each variable the expected value is provided, (e.g. the expected carrier's search time in $H$ is almost 2 days). 
Note that we only allow for search in the location, whereas in reality carriers are able to contact customers before they arrive in the location. Hence, we have chosen longer search times than those reported in surveys (Meelker, 2006).

Table 4: Endogenous variables for the reference case of the matching model

\begin{tabular}{lll}
\hline Variable & Value & Description \\
\hline$p_{H}$ & $€ 5,785.21$ & Price from $H$ to $L$ \\
$p_{L}$ & $€ 1,043.66$ & Price from $L$ to $H$ \\
$\mu_{L}$ & 0.1904 & Fraction of arriving carriers that search in $L$ \\
$C$ & 399.9346 & Total number of carriers present in the market \\
Carriers' search time in $H$ & 1.9211 days & Calculated as 365.25 days $/ \theta_{H} q_{H}$ \\
Carriers' search time in $L$ & 2.5434 days & Calculated as 365.25 days $/ \theta_{L} q_{L}$ \\
Customers' search time in $H$ & 9.7120 days & Calculated as 365.25 days $/ q_{H}$ \\
Customers' search time in $L$ & 12.751 days & Calculated as 365.25 days $/ q_{L}$ \\
Number of matches per year in $H\left(Q_{H}\right)$ & $15,043.20$ & Calculated as $\phi C_{S H}^{\gamma} N_{H}^{\delta}$ \\
Number of matches per year in $L\left(Q_{L}\right)$ & 2864.50 & Calculated as $\phi C_{S L}^{\gamma} N_{H}^{\delta}$ \\
Single-trip moving time & 3.6525 days & Calculated as 365.25 days $/(2 \lambda)$ \\
Single-trip moving cost & $€ 2,498.75$ & Calculated as $m c /(r+\lambda)$ \\
Carriers' search cost in $H$ & $€ 788.96$ & Calculated as $s c / \theta_{H} q_{H}$ \\
Carriers' search cost in $L$ & $€ 1,044.53$ & Calculated as $s c / \theta_{L} q_{L}$ \\
\hline
\end{tabular}

From Table 5, as $S_{L}=M_{L}$, it can be seen that the Imbalanced Equilibrium arises. We have also varied $N_{H}$ (see section 3.3) but it appears that the Balanced Equilibrium only arises if $N_{H}$ is extremely close to $N_{L}$.

In agreement with Theorem 1 we see that the backhaul price $p_{L}$ is positive and substantial, $p_{L}$ is $18 \%$ of $p_{H}$, even though $81 \%$ of carriers return empty to $H\left(\mu_{L}=0.19\right)$. In addition, due to the increasing returns to scale property of the matching function, carriers' expected search times in $\mathrm{L}$ are about $31 \%$ longer than in $\mathrm{H}$, implying a difference in search cost of $€ 256$ (=€1,045-€789).

Numerical analysis is now done by varying parameters and comparing the outcomes with the outcomes of the reference case. Below we distinguish three cases which correspond to the changing of speed $\lambda$, demand imbalance $N_{H}$ ( $N_{L}$ is kept constant), and the value of the transported good $J$ respectively. 
Note that the number of carriers, $C$, in the market is almost identical to the number of carriers in $H, N_{H}$. This is pure accidental. Later on, we will see that $\mathrm{C}$ will be substantially different from $N_{H}$.

Table 5: Endogenous variables for the reference case of the matching model

\begin{tabular}{lll}
\hline Variable & Value & Description \\
\hline$S_{H}$ & $€ 0$ & Asset value of searching for a carrier in $H$ \\
$M_{H}$ & $-€ 4,996.25$ & Asset value of moving for a carrier in $H$ \\
$S_{L}$ & $-€ 2.498 .75$ & Asset value of searching for a carrier in $L$ \\
$M_{L}$ & $-€ 2.498 .75$ & Asset value of moving for a carrier in $L$ \\
$V_{H}$ & $€ 93,425.82$ & Asset value of searching for a customer in $H$ \\
$V_{L}$ & $€ 97,912.68$ & Asset value of searching for a customer in $L$ \\
$q_{H}$ & 37.6080 & Rate of finding a carrier for a customer in $H$ \\
$q_{L}$ & 28.6450 & Rate of finding a carrier for a customer in $L$ \\
$\theta_{H}$ & 5.0554 & Market tightness \\
$\theta_{L}$ & 5.0133 & Market tightness \\
$u_{H}$ & 0.1978 & Fraction of total carriers that search in $H$ \\
$u_{L}$ & 0.0499 & Fraction of total carriers that search in $L$ \\
$v_{H}$ & 1.0002 & Ratio of customers in $H$ to total carriers \\
$v_{L}$ & 0.2500 & Ratio of customers in $L$ to total carriers \\
$C_{S H}$ & 79.1234 & Number of carriers that search in $H$ \\
$C_{S L}$ & 19.9470 & Number of carriers that search in $L$ \\
$C_{M}$ & 300.8641 & Number of carriers that are on their way moving \\
\hline
\end{tabular}

\subsection{Delays in transport caused by climate change}

Due to climate change it is expected that the Rhine river will be blocked more often due to too high water levels in winter and too low water levels in summer (see e.g. Jonkeren et al., 2007). So speeds of transportation will be reduced. In our model, the effects of a reduction in speed can be analysed by a decreasing $\lambda$. We decrease $\lambda$ from 50 (the reference case) to 45 as well as to 40. It appears that the type of equilibrium (Imbalanced Equilibrium) does not change. The results for the endogenous variables (the same variables as in Table 4) can be found in Table 6. 
Table 6: Effects of variations in the round trip rate $\lambda$

\begin{tabular}{llll}
\hline Variable & Reference Case & Case 1 & Case 2 \\
\hline$\lambda \quad$ (exogenous) & 50 & 45 & 40 \\
$p_{H}$ & $€ 5,785.21$ & $€ 6,339.60$ & $€ 7,032.44$ \\
$p_{L}$ & $€ 1,043.66$ & $€ 1,043.60$ & $€ 1,043.53$ \\
$\mu_{L}$ & 0.1904 & 0.1905 & 0.1907 \\
$C$ & 399.9346 & 433.1046 & 474.5143 \\
Carriers' search time in $H$ & 1.9211 days & 1.9204 days & 1.9195 days \\
Carriers' search time in $L$ & 2.5434 days & 2.5435 days & 2.5436 days \\
Customers' search time in $H$ & 9.7120 days & 9.7175 days & 9.7243 days \\
Customers' search time in $L$ & 12.7509 days & 12.7502 days & 12.7493 days \\
Number of matches per year in $H$ & $15,043.20$ & $15,034.78$ & $15,024.24$ \\
Number of matches per year in $L$ & $2,864.50$ & $2,864.66$ & $2,864.85$ \\
Single-trip moving time & 3.6525 days & 4.0583 days & 4.5656 days \\
Single-trip moving cost & $€ 2,498.75$ & $€ 2,776.24$ & $€ 3,123.05$ \\
Carriers' search cost in $H$ & $€ 788.96$ & $€ 788.67$ & $€ 788.29$ \\
Carriers' search cost in $L$ & $€ 1,044.53$ & $€ 1,044.57$ & $€ 1,044.62$ \\
\hline
\end{tabular}

The results of a lower speed, captured by $\lambda$, implies, of course, a substantially longer expected transport time per trip and a higher transport cost. It appears that both customers' and carriers' search times and number of matches are hardly affected however, so the increase in transport cost is borne entirely by customers in $H$, which pay higher prices. So, customers in the high demand location $H$ will be negatively affected ( $V_{H}$ will decrease), while no such thing happens for customers in the low demand location. Note that $p_{L}$ is hardly affected. The implication is for example that customers in the harbour of Rotterdam (the high demand location) will be affected, whereas customers in Germany (the low demand location) will hardly be affected. Hence, the welfare losses of changes in water levels will be borne almost entirely by consumers that have a demand for the products coming from Rotterdam.

\subsection{Stronger imbalance in demand}

We present Case 3 and Case 4 to see how sensitive our outcomes are for variations in the imbalance between the two markets. $N_{L}$ is kept constant at 100 in this analysis, and $N_{H}$ is set 
from 400 in the reference case to 800 and 1600 respectively. The results can be found in Table 7.

Table 7: Effects of variations in the number of customers in $H, N_{H}$

\begin{tabular}{llll}
\hline Variable & Reference Case & Case 3 & Case 4 \\
\hline$N_{H} \quad$ (exogenous) & 400 & 800 & 1600 \\
$p_{H}$ & $€ 5,785.21$ & $€ 5,683.18$ & $€ 5,594.33$ \\
$p_{L}$ & $€ 1,043.66$ & $€ 1,043.66$ & $€ 1,043.66$ \\
$\mu_{L}$ & 0.1904 & 0.0829 & 0.0361 \\
$C$ & 399.9346 & 869.5951 & $1,925.2275$ \\
Carriers' search time in $H$ & 1.9211 days & 1.6727 days & 1.4563 days \\
Carriers' search time in $L$ & 2.5434 days & 2.5434 days & 2.5434 days \\
Customers' search time in $H$ & 9.7120 days & 8.4531 days & 7.3575 days \\
Customers' search time in $L$ & 12.7509 days & 12.7509 days & 12.7509 days \\
Number of matches per year in $H$ & $15,043.20$ & $34,567.39$ & $79,429.15$ \\
Number of matches per year in $L$ & $2,864.50$ & $2,864.50$ & $2,864.50$ \\
Carriers' search cost in $H$ & $€ 788.96$ & $€ 686.93$ & $€ 598.08$ \\
Carriers' search cost in $L$ & $€ 1,044.53$ & $€ 1,044.53$ & $€ 1,044.53$ \\
\hline
\end{tabular}

When we analyse a more imbalanced demand between locations, we see that most variables are rather insensitive to stronger imbalance. Especially $p_{L}$ is seen to be insensitive. The decrease in $p_{H}$ can be explained by the increasing returns to scale matching function. A higher demand in $H$ means that matches are easier formed. This implies lower search times, lower search cost and therefore lower freight prices in $H$.

\subsection{Different values of the transported good}

We explore now the sensitivity of the outcomes for the value of the transported good. The resulting output for this analysis can be found in Table 8 .

The outcomes make sense and are easy to interpret. As there becomes more surplus available with an increasing value of the good, (a higher $J$ ), carriers will in first instance also profit from this surplus due to their market power. However, due to the free-entry condition, more carriers 
Table 8: Effects of variations in the value of the transported good (parameter $J$ )

\begin{tabular}{llll}
\hline Variable & Case 5 & Reference Case & Case 6 \\
\hline$J \quad$ (exogenous) & $€ 50,000$ & $€ 100,000$ & $€ 200,000$ \\
$p_{H}$ & $€ 5,757.95$ & $€ 5,785.21$ & $€ 5,835.88$ \\
$p_{L}$ & $€ 1,007.89$ & $€ 1,043.66$ & $€ 1,110.18$ \\
$\mu_{L}$ & 0.1905 & 0.1904 & 0.1903 \\
$C$ & 376.1499 & 399.9346 & 446.0249 \\
Carriers' search time in $H$ & 1.8547 days & 1.9211 days & 2.0445 days \\
Carriers' search time in $L$ & 2.4562 days & 2.5434 days & 2.7055 days \\
Customers' search time in $H$ & 10.2381 days & 9.7120 days & 8.8464 days \\
Customers' search time in $L$ & 13.4358 days & 12.7509 days & 11.6223 days \\
Number of matches per year in $H$ & $14,270.22$ & $15,043.20$ & $16,515.15$ \\
Number of matches per year in $L$ & $2,718.48$ & $2,864.50$ & $3,142.65$ \\
(Expected) search cost in $H$ for a carrier & $€ 761.70$ & $€ 788.96$ & $€ 839.62$ \\
(Expected) search cost in $L$ for a carrier & $€ 1008.73$ & $€ 1,044.53$ & $€ 1,111.10$ \\
\hline
\end{tabular}

will enter until this advantage disappears. Nevertheless, higher prices $p_{H}$ and $p_{L}$ result. Due to an increase in $J$, however, the increase in $p_{L}$ exceeds the increase in $p_{H}$. For example, when $\mathrm{J}=200,000$ (case 6 ), $p_{H}$ increases by $0.88 \%$ and $p_{L}$ by $6.37 \%$. This may be loosely explained as follows. As carriers have market power, the increase in the value of the transported good will result in higher freight prices, through the increase the total surplus. Since we are in the Imbalanced Equilibrium, the increase in freight prices can only imply an increased search time for carriers and the round trip transport cost remains still part of $p_{H}$. We have an increasing returns to scale matching function and the market in $L$ is smaller, therefore the search time in $L$ is expected to increase more than in $H$. This explains the faster increase of $p_{L}$ compared with $p_{H}$.

\section{Conclusion}

In this paper we modeled the backhaul problem by means of a matching model in a twolocation transport framework. We were motivated by observations that competitive models do not adequately explain positive backhaul prices. In particular, according to the competitive model, backhaul freight prices drop to zero when the quantities transported between locations differ, so a proportion of the carriers return without freight from low demand locations. Costs 
of search and waiting may explain this phenomenon, but it would be ad-hoc to incorporate search costs exogenously in the competitive model. Therefore we develop a model where search times and therefore search costs are determined endogenously. We demonstrate how these search times vary with certain essential exogenous parameters, such as transport cost.

We presented a two-location transport model with which we studied backhaul pricing. Similar to the competitive model, it turned out that we have to distinguish between two kinds of equilibria: (1) the Balanced Equilibrium, so carriers transport freight in both directions and, (2) the Imbalanced Equilibrium: some carriers move without from the low demand location. The Imbalanced Equilibrium is the more interesting one from an empirical perspective. Indeed, given a wide range of parameters, we end up in the Imbalanced Equilibrium.

In the Imbalanced Equilibrium, the round trip transport cost is fully borne by the customers in the high demand location. However, positive backhaul prices result due to carriers' compensation for expected search time. Hence, we have addressed the above mentioned limitation of the competitive model. To study the sensitivity of the search times to a few essential parameters, we performed a comparative statics analysis, in which the chosen parameter set was taken from the inland navigation market on the Rhine river in Western Europe.

First, we studied the effect of reduction in travel speed due to changes in water-levels, e.g. as a result of climate change, leading to higher trip durations. The interesting thing to note was that the customers, in the high demand location will pay for the increased costs of transport. Hence, the welfare losses will be borne by consumers that demand goods from the high demand region.

Second we investigated the consequences of stronger imbalances in demand by increasing fronthaul demand and keeping backhaul demand constant. Backhaul prices turned out to be insensitive to this kind of demand increase.

And third we studied sensitivity to the value of the transported good. Increasing the value of the good means that the customers' value of transporting the good increases. So waiting is more costly for the customers. As a result, carriers are able to increase freight prices, the effect being larger for backhaul prices.

We end with a few recommendations for further research. First it might be an improvement to allow carriers to search for contact with customers while they are moving. Second, a more realistic production process for transport demanding customers could be 
modeled. And third, empirical research is needed to test the main theoretical results in the current paper. 


\section{References}

Arnott, R. (1996) Taxi travel should be subsidized, Journal of Urban Economics 40, pp. 316-333

Bellman, R (1952) On the theory of dynamic programming, Proceedings of the National Academy of Sciences, pp. 503-515

Binmore, K.G. Rubinstein, A., Wolinsky, A. (1986) The Nash bargaining solution in economic modelling, Rand Journal of Economics 17, pp. 176-188

Boyer, K.D. (1998) Principles of Transportation Economics, pp. 252-257, Massachussets, Addison-Wesley Reading

Felton, J.R. (1981) The impact of rate regulation upon ICC-regulated truck back hauls, Journal of Transport Economics and Policy 15, (3), pp. 253-267

Jonkeren, O., Rietveld, P., van Ommeren, J. (2007) Climate change and inland waterway transport; welfare effects of low water levels on the river Rhine, Journal of Transport Economics and Policy 41 (3) pp. 387-411

Lagos, R.A. (1996) An alternative approach to market frictions: an application to the market for taxicab rides, CARESS Working Paper 96-09, University of Pennsylvania, November 1996

Lee, J.W., McKibbin, W.J., Park, Y.C. (2006) Transpacific trade imbalances: causes and cures, The World Economy 29 (3), pp. 281-303.

Meelker, C. (2006), The Dutch Inland Waterway Transport Market: The Backhaul Problem, Master Thesis, Vrije Universiteit, Amsterdam, The Netherlands

Mortensen, D.T., Pissarides, C.A. (1999) Unemployment responses to 'skill-biased' technology shocks the role of labour market policy, The Economic Journal 109, pp. 242-265.

Van Ommeren, J., Rietveld, P. (2002) Commuting and reimbursement of residential relocation costs, Journal of Transport Economics and Policy 41(1), pp. 51-73

Pigou, A.C., Taussig, F.W. (1913) Railway rates and joint costs, The Quarterly Journal of Economics 27 (3), pp. 687-694

Pissarides, C.A. (1994) Search unemployment with on-the-job search, The Review of Economic Studies 61 (3), pp. 457-475 
Pissarides, C.A. (2000) Equilibrium Unemployment Theory, Massachussets Institute of Technology

Rietveld, P., Roson, R. (2002) Direction dependent prices in public transport: A good idea? The back haul pricing problem for a monopolistic public transport customer, Transportation 29, pp. 397-417

Statistics Netherlands, The Hague, Netherlands, www.cbs.nl

Warren Jr., R.S. (1996) Returns to scale in a matching model of the labor market, Economic Letters 50, pp. 135-142

Wheaton, W.C. (1990) Vacancy, search, and prices in a housing market matching model, The Journal of Political Economy 98 (6), pp. 1270-1292

Wilson, W.W. (1987) Transport markets and customer behavior: the backhaul problem, Journal of the Transportation Research Forum 28, pp. 325 - 333 


\section{Appendix A - Restrictions on Asset Values}

We would like to prove that $S_{H}>M_{H}$ or $S_{L}>M_{L}$. We will prove this by contradiction.

Suppose first that $S_{H} \leq M_{H}$ and $S_{L} \leq M_{L}$. Note that $S_{H}=0$, which implies that $M_{H} \geq 0$.

Equation (2) implies then that for $i=H, L$ :

$$
r M_{i}=-m c+2 \lambda\left(M_{j}-M_{i}\right), \quad j \neq i,
$$

which implies that $M_{H}=M_{L}=-m c / r<0$. This is inconsistent with $M_{H} \geq 0$.

Hence, we have established $S_{H}>M_{H}$ or $S_{L}>M_{L}$.

\section{Appendix B - $\quad$ Derivation of Equations (5) and (6)}

Independent whether the type of equilibrium is Balanced or Imbalanced, $S_{H}>M_{H}$, $S_{L} \geq M_{L}$ and $S_{H}=0$. The Bellman equations (1) and (2) for $i=L, H$ can then be written as:

$$
\begin{aligned}
& 0=-s c+\theta_{H} q_{H}\left(M_{H}+p_{H}\right) \\
& r M_{H}=-m c+2 \lambda\left(S_{L}-M_{H}\right) \\
& r S_{L}=-s c+\theta_{L} q_{L}\left(M_{L}-S_{L}+p_{L}\right) \\
& r M_{L}=-m c+2 \lambda\left(-M_{L}\right)
\end{aligned}
$$

These four equations imply that:

$$
\begin{aligned}
& p_{H}=\frac{s c}{\theta_{H} q_{H}}+\frac{m c}{r+2 \lambda}-\frac{2 \lambda}{r+2 \lambda} S_{L} \\
& p_{L}=\frac{s c}{\theta_{L} q_{L}}+\frac{m c}{r+2 \lambda}+\frac{\theta_{L} q_{L}+r}{\theta_{L} q_{L}} S_{L}
\end{aligned}
$$

Equation (B5) corresponds with equation (5) in the text, and (B6) corresponds with (6). 


\section{Appendix C - Proof of Theorem 1 (for the Imbalanced Equilibrium)}

\section{Theorem 1.}

In a matching model for a two-location market the backhaul price $p_{L}$ will be strictly positive, under the condition that the discount rate is strictly positive $(r>0)$.

\section{Proof.}

In the Imbalanced Equilibrium we know from (7) that $p_{L}=\frac{s c}{\theta_{L} q_{L}}$, and from (15) that $p_{L}=\frac{\beta(r J+\kappa)}{r+(1-\beta) q_{L}}$. Setting these two equal and using $\theta_{L} q_{L}=\alpha m\left(1, \theta_{L}\right)$, and therefore $q_{L}=\alpha m\left(\frac{1}{\theta_{L}}, 1\right)=\frac{\alpha m\left(1, \theta_{L}\right)}{\theta_{L}}$, one obtains after some manipulation:

$$
\theta_{L} q_{L}=\frac{\operatorname{scr} \theta_{L}}{\beta(r J+\kappa) \theta_{L}-s c(1-\beta)}
$$

In the remainder of the proof we use the assumption that $q_{L}>0$. This is justified as we focus now on the Imbalanced Equilibrium. So we already assume that transport exists from the low demand location to the high demand location. This implies a positive customers contact rate $q_{L}$. From (7), (15), $q_{L}>0$, and the condition $r>0$, one obtains:

$$
\theta_{L}=\frac{s c}{p_{L} q_{L}}=\frac{s c\left(r+(1-\beta) q_{L}\right)}{q_{L} \beta(r J+\kappa)}=\frac{s c r}{q_{L} \beta(r J+\kappa)}+\frac{s c(1-\beta)}{\beta(r J+\kappa)}>\frac{s c(1-\beta)}{\beta(r J+\kappa)}
$$

Therefore $\theta_{L} q_{L}$ in $(C 1)$ is finite. We know from (7) that, in the Imbalanced Equilibrium, $p_{L}=\frac{s c}{\theta_{L} q_{L}}$. Therefore $p_{L}>0$ 